P14

EXAMINING THE OUTCOMES OF A PLEURAL DISEASE CLINIC

${ }^{1}$ DT Whitehall, ${ }^{2} \mathrm{JB}$ McCafferty. ${ }^{1}$ The University of Edinburgh, Edinburgh, UK; ${ }^{2} \mathrm{NHS}$ Lothian, Edinburgh, UK

10.1136/thoraxjnl-2016-209333.157

Background Pleural effusions, the result of the accumulation of fluid in the pleural space, are a common medical problem. Patients with symptoms of cough and breathlessness with associated signs and chest $\mathrm{x}$-ray (CXR) changes suggestive of a pleural effusion commonly present acutely to the medical assessment unit or to the respiratory outpatient department. Pleural effusions may also develop during admission. Advances in the availability of pleural diagnostic techniques, including thoracic ultrasound, thoracocentesis kits, and medical thoracoscopy means that pleural expertise can be concentrated in a dedicated clinic with the aim of facilitating ambulatory care, reducing hospital admissions and rationalising the use of laboratory services. This study aimed to assess the impact of a dedicated pleural clinic on these factors.

Methods A retrospective analysis of the hospital electronic patient records was carried out on patients attending the pleural clinic from 2014 to 2016. 146 patients were identified. Hospital admission data was also evaluated to assess inpatient admission for pleural effusion before and after the pleural clinic was instituted. In addition, quantification of laboratory samples sent preand post-pleural clinic was carried out.

Results Malignant disease was diagnosed in $44 \%$ of cases versus $46 \%$ for benign disease. A $29 \%$ reduction in ward admissions for pleural effusion was seen over 2 years. With a median length of stay of 5 days this resulted in 175 bed days saved in 205 with an associated annual cost saving of approximately $£ 87,000$. The number of diagnostic samples sent for cytology dropped by $11 \%$ following the introduction of the pleural clinic with estimated annual cost savings of around $£ 3000$.
Conclusions The introduction of a pleural clinic is not only cost effective in reducing hospital admissions and optimising diagnostic costs but also improves the patient journey by facilitating ambulatory care wherever possible.

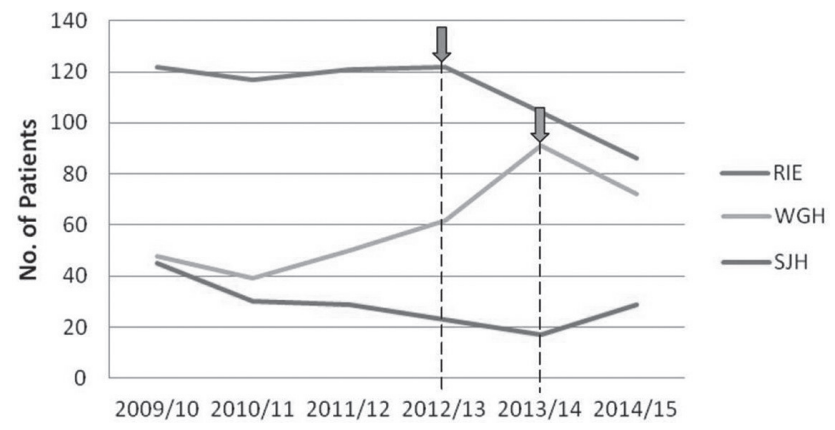

Abstract P14 Figure 1 Pleural effusion inpatient admissions at three different hospitals.

Arrows $=$ Pleural clinic established at hospital

\section{P15 A PILOT STUDY OF A DEDICATED BALLOONED INTERCOSTAL DRAIN}

S Ross, H Ali, L Allsop, NJ Ali, SV Kemp. Sherwood Forest Hospitals NHS Foundation Trust, Sutton-in-Ashfield, UK

\subsection{6/thoraxjnl-2016-209333.158}

Introduction Intercostal tube drainage of pleural air or fluid is an essential tool in the management of respiratory patients. A common complication of drain insertion is accidental removal of the drain, usually as a result of inadequate securing techniques, with rates of up to $21 \%$ quoted in the literature. ${ }^{1,2}$ This often results in the need for further pleural procedures (including drain re-siting), with associated additional risk to the patient and an increase
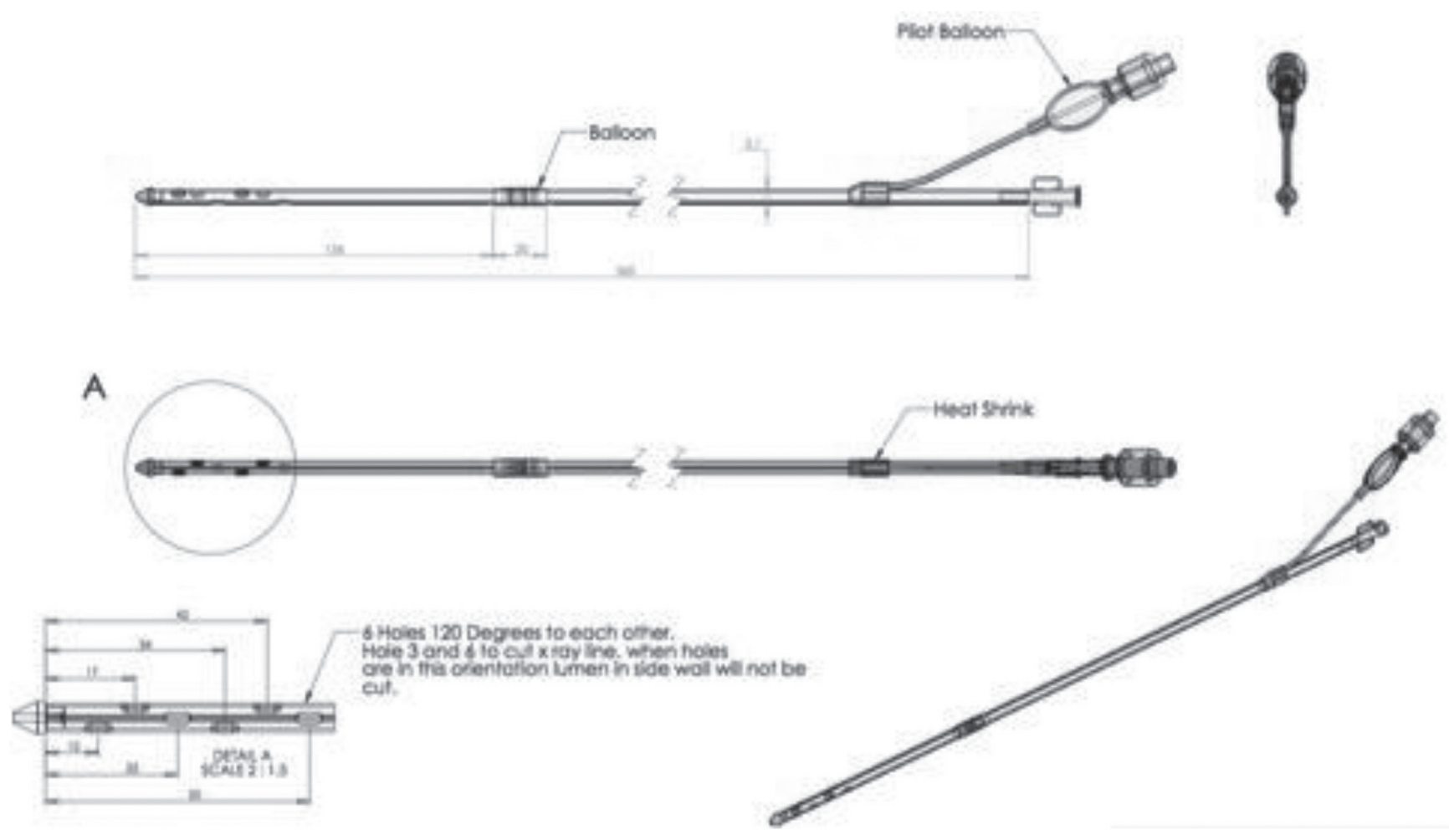

Abstract P15 Figure 1 
in health care costs. One suggested method to reduce premature drain removal is to use intercostal drains with ballooned tips. These would provide a relatively atraumatic physical obstruction to the thoracostomy site, whilst being easy to use as stitching or extensive taping may not be required.

Methods We conducted a pilot study of a dedicated $16 \mathrm{~F}$ ballooned intercostal drain (Rocket Medical; Figure 1) to assess its safety and feasibility, and to give an indication as to whether a reduction in accidental early drain removal could be achieved. Drains were inserted under ultrasound guidance using the Seldinger technique and secured with dedicated dressings. Skin sutures were not applied. Pain scores were collected from a subset of 11 patients using a visual analogue scale (VAS) from 0 (no pain) to 10 (worst pain imaginable) at insertion, $24 \mathrm{~h}$ post-insertion, and drain removal to ensure pleural irritation was not prohibitive.

Results Twenty patients requiring intercostal tube drainage as an in-patient for pleural effusion were recruited from a single hospital site. Of the drains inserted, 1/20 (5\%) was prematurely dislodged., comparing favourably with the literature. Inspection of the device showed that the balloon had become partially unglued from the drain, hence deflation. The patient did not require further pleural procedures. No other drain-related adverse events were recorded. The drains were generally well tolerated, with median(range) pain scores at insertion, 24 hours, and removal of $1(0-7), 3(0-8)$, and $2(0-7)$.

Conclusion The use of a dedicated ballooned intercostal drain is safe and feasible, and may reduce the need for drain re-siting. A larger randomised trial is planned.

\section{REFERENCES}

1 Corcoran JP, et al. Pleural procedural complications: prevention and management. J Thorac Dis 2015;7(6):1058-67.

2 Horsley $A$, et al. Efficacy and complications of small-bore, wire-guided chest drains. Chest 2006;130:1857-63.

\section{Lung Cancer Investigations}

\section{P16 RADIAL EBUS BIOPSY WITH GUIDE SHEATH FOR PERIPHERAL PULMONARY LESIONS}

K Srikanthan, C Drouot, B Sukumaran, V Johnson, M Walshaw, K Mohan. Liverpool Heart and Chest Hospital, Liverpool, UK

\subsection{6/thoraxjnl-2016-209333.159}

Introduction The aim of lung cancer screening programs is the detection of early lung cancer, which may appear as small peripheral pulmonary lesion (PPL). Although radial EBUS guided biopsy is recommended by NICE to obtain tissue diagnosis in PPL which cannot be seen by conventional bronchoscopy, there is a paucity of published data within the UK regarding this technique. We looked at our 4-year radial EBUS results at our tertiary centre.

Methods We reviewed 71 consecutive patients who underwent radial EBUS guided biopsy for investigation of a PPL, performed using a guide sheath (K201 or K203) by a consultant operator assisted by a respiratory trainee. We assessed the diagnostic rate, yield from sampling techniques (biopsy vs brushings), whether the diagnosis correlated with other procedures/management, and any complications.

Results Mean patient age was 70 years (range 44-89), 38 female, and $41(58 \%)$ had undergone 1 or more unsuccessful investigations at their local hospital. We were able to visualise the lesion by ultrasound leading to subsequent sampling in 62/71 (87\%) patients. A diagnosis of malignancy was confirmed in 41/62 (66\%) patients. Of the 21 biopsies which did not demonstrate malignancy, 11 were subsequently shown to have cancer (false negatives-pathological diagnosis by other methods or clinicalradiological diagnosis or awaiting follow up) but were 10 true negative (resolution or 2 year stability on CT scan). The yield for malignancy was superior with brushings (88\%) compared to biopsy (73\%). The overall sensitivity for cancer was $72 \%$ ( $\mathrm{N}=71$, whole cohort) and $82 \%(\mathrm{~N}=62$, lesion was visualised by ultrasound) respectively. Two patients developed a pneumothorax which did not require intervention.

Conclusions Radial EBUS is a safe and effective technique in obtaining tissue diagnosis in PPL not amenable to other biopsy methods. We visualised the lesion in $87 \%$ of patients and our diagnostic yield for malignancy is similar to current standards. Radial EBUS should take a more prominent role in diagnostic pathways for PPL within the UK, particularly in the context of future lung cancer screening programmes.

\section{P17 A RETROSPECTIVE ANALYSIS COMPARING THE USE OF PROCORE WITH STANDARD FINE NEEDLE ASPIRATION IN ENDOBRONCHIAL ULTRASOUND GUIDED TRANSBRONCHIAL NEEDLE ASPIRATION (EBUS-TBNA)}

DJ McCracken, TE McManus, A Shamboul. South West Acute Hospital, Enniskillen, UK

10.1136/thoraxjnl-2016-209333.160

Endobronchial ultrasound has become first line in the investigation of mediastinal lesions suspicious for malignancy in keeping with NICE guidelines, however needle size and type, along with number of passes required to maximise diagnostic sensitivity remains unclear.

Previous meta-analyses, the largest of which included 576 patients, ${ }^{1}$ have compared the use of ProCore with standard fine needle aspiration in the assessment of pancreatic masses with differences noted only in the number of passes required.

We aim to assess whether a ProCore needle improves diagnostic sensitivity in EBUS-TBNA.

Complete follow up data regarding all 235 patients undergoing EBUS-TBNA in a district general hospital has been collected since the service's inception in 2012. Results were collated and retrospectively analysed allowing for calculation of test sensitivity and specificity. Comparison was then made between procedures where standard fine needle aspiration was performed and those using a ProCore needle.

Overall sensitivity of EBUS-TBNA was shown to be $85 \%$ with a specificity of $100 \%$ in keeping with quoted figures from other centres. Standard fine needle aspiration produced a sensitivity of $77 \%(85 / 110)$ versus ProCore sensitivity of 92\% (115/125) with a $\mathrm{p}$ value of 0.0016 .

$30 \%(33 / 110)$ of patients undergoing standard fine needle aspiration required an appropriate crossover technique such as mediastinoscopy or CT guided FNA in order to either obtain or confirm the diagnosis compared with 15\% (19/125) of the ProCore group with a $\mathrm{p}$ value of 0.0064 .

Our retrospective analysis shows a statistically significant difference in the diagnostic sensitivity of sampling mediastinal lymphadenopathy using a ProCore needle compared with standard fine needle aspiration. It also shows that a significantly fewer number of patients required further procedures in order to obtain or confirm the diagnosis. This could potentially be confounded by the retrospective nature of the study design, however due to 